\title{
Multi-Step in 3D Spin Crossover Nanoparticles Simulated by an Ising Model Using Entropic Sampling Monte Carlo Technique
}

\author{
Catalin Jureschi ${ }^{1,2}$, Jorge Linares ${ }^{3, *}$, Aurelian Rotaru ${ }^{2}$ and Yann Garcia ${ }^{4, *}$ \\ 1 LISV, Université de Versailles Saint-Quentin-en-Yvelines, Université Paris Saclay, 78140 Velizy, France; \\ catalin.jureschi@gmail.com \\ 2 Faculty of Electrical Engineering and Computer Science \& Research Center MANSiD, \\ Stefan cel Mare University, Suceava 720229, Romania; aurelian.rotaru@gmail.com \\ 3 GEMaC, Université de Versailles Saint-Quentin-en-Yvelines, CNRS-UVSQ (UMR 8635), \\ Université Paris Saclay, 78035 Versailles Cedex, France \\ 4 Institute of Condensed Matter and Nanosciences, Molecules, Solids and Reactivity (IMCN/MOST), \\ Université catholique de Louvain, Place L. Pasteur 1, 1348 Louvain-la-Neuve, Belgium \\ * Correspondence: jlinares1949@yahoo.fr (J.L.); yann.garcia@uclouvain.be (Y.G.); Tel.: +33-13925466 (J.L.) \\ Academic Editors: Guillem Aromí and José Antonio Real \\ Received: 25 January 2016; Accepted: 22 February 2016; Published: 1 March 2016
}

\begin{abstract}
There are currently extended experimental and theoretical developments of spin crossover nanomaterials, in particular based on coordination polymers for the design of smart applications. In this context, we have reproduced a three step thermal transition in a cubic spin crossover nanomaterial with a system dimension of $5 \times 5 \times 5$ metallic centers. For this purpose, we have calculated, using Monte Carlo Entropic Sampling technique, the density of states of all possible system configurations. In order to take into account the local environment, we have included an additional interaction term in the standard Ising like model. We have then analyzed the role of this new interaction as well as the system size effect variation (from $4 \times 4 \times 4$ to $6 \times 6 \times 6$ metallic centers). Comparison with a 2D SCO system shows that the spin transition still proceeds in three steps but is no longer hysteretic.
\end{abstract}

Keywords: spin crossover; Ising model; Monte Carlo; metal organic frameworks

\section{Introduction}

The potential applications of spin crossover (SCO) materials as temperature and pressure sensors [1-3], actuators, memory devices or electrical switches [4] and their possible spin state control at the molecular level are some important assets of these fascinating switching materials. SCO coordination compounds display a central metallic ion in an octahedral environment. Considering that the central ion is an iron atom, thus, as a function of the ligand field strength, the six electron of the Fe(II) ion can occupy the $3 \mathrm{~d}$ orbitals in two different ways giving rise to two different stable states: a diamagnetic low-spin (LS) state and a paramagnetic high-spin (HS) state (Figure 1). The transition between the two spin states can be induced by applying external perturbations such as: a temperature variation, external pressure, light irradiation, magnetic or electrical field or by a chemical adsorption-desorption phenomenon [5-10]. In the last years, several experimental studies concerning the role of cooperativity in SCO compounds have been reported $[2,3,11-19]$ in this context giving a great attention to stepwise transitions, in particular those presenting a two-step SCO behavior. It is nowadays commonly accepted that this type of switching phenomenon results from a synergistic effect between intramolecular interactions resulting from the strong bonding between metallic centres favoring an antiferromagnetic-like state (LS-HS-LS-HS,...) and intermolecular interactions of elastic 
origin favoring domains with the same spin sate (ferromagnetic-like). The origin of the recently discovered three-step transitions [11-13] is however far from being understood, with little theoretical studies at present [20-25]. Indeed, various models and theoretical techniques have been developed to predict precisely the influence of perturbation factors in the evolution of thermal SCO behavior. One of the most useful theoretical tool is presumably the Ising like model which was introduced by Wajnflasz and Pick in 1971 [26]. This model was considering only the short-range interaction between switching sites. With such a limitation, this model was unable for instance to reproduce hysteretic spin transition in 1D SCO systems. Later, Bousseksou et al. [27] reported the modelisation of a two-step spin conversion using two "antiferromagnetically" coupled sublattices, in the mean-field approximation. Linares et al. [28] improved this model by introducing a long range interaction in the Hamiltonian, which they successfully applied to 1D SCO chains. Under these conditions hysteretic spin transitions or two step spin transitions were covered [29]. In 2015, Chiruta et al. [30] introduced a new term in the Hamiltonian, by considering a new elastic interaction between edge metallic centers with their local environment. This step allowed reproducing multi-step transitions for 1D SCO systems. In addition, Chiruta et al. [31] performed theoretical studies that highlighted the role of short- and long-range interactions for system metallic centers with periodic conditions. They showed that the long range interaction parameter is the basis of hysteretic spin transitions, while the short range interaction parameter is the basis of two step transitions. It was also demonstrated that the edge effect is at the origin of multi-step transitions, even if the metallic centers are fixed in the HS state [32] or are active metallic centers that interact with their local environment [30,33]. Other important theoretical results were reported considering systems architecture [34] or molecule size effects [31,35,36]. In the current account, we consider the influence of interaction between edge metallic centers with their local environment for different architectures in 2D and 3D SCO materials.

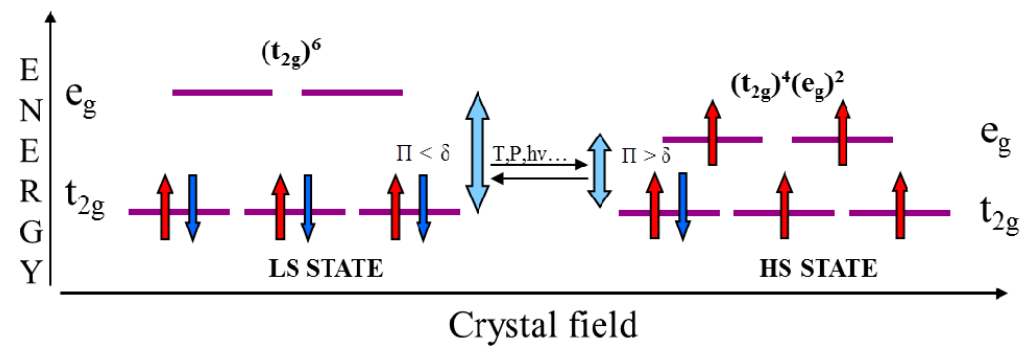

Figure 1. Electronic diagram of the high-spin (HS) and low-spin (LS) states for a Fe(II) ion in an octahedral ligand field.

\section{Materials and Methods}

\subsection{Ising Like Model}

To simulate the interaction between edge metallic centers with the environment, we have used the following Hamiltonian:

$$
H=\frac{\Delta-k_{B} T \ln g}{2} \sum_{i=1}^{N} \sigma_{i}-G \sum_{i=1}^{N} \sigma_{i}<\sigma>-J \sum_{\langle i, j\rangle} \sigma_{i} \sigma_{j}-L \sum_{i=1}^{M} \sigma_{i},
$$

where $\sigma$ is a fictitious spin operator which can take the value -1 when the metallic center is in the LS state and the value +1 when the metallic center is in the HS state, $\Delta$ is the energy gap, $k_{B}$ is the Boltzmann constant, $g=g_{H S} / g_{L S}$ represents the ratio of degeneracies of the two spin states, HS and low-spin (LS), $N$ is the number of metallic centers, $J$ and $G$ represent short- and long-range interactions, $L$ is the interaction parameter of $M$ edge metallic centers with their local environment. Since from experimental results it is clear that the environment favors the HS state, we have considered in this contribution a positive value of $L$. 
Dimensionless macroscopic variables were also used:

$$
\begin{gathered}
m=\sum_{i=1}^{N} \sigma_{i}, \\
s=\sum_{<i, j>} \sigma_{i} \sigma_{j}, \\
c=\sum_{k=1}^{M} \sigma_{k}^{\prime},
\end{gathered}
$$

where $\sigma_{k}^{\prime}$ represents the metallic centers at the "surface" (edge-metallic centers).

The Hamiltonian of the system can be written:

$$
H=\left(\frac{\Delta-k_{B} T \ln g}{2}-G\langle\sigma\rangle\right) m-J s-L c,
$$

The HS fraction, $n_{H S}$, is given by:

$$
n_{H S}=\frac{<\sigma>+1}{2},
$$

where, the average fictitious magnetization can be written as:

$$
<\sigma>=\frac{\sum_{i=1, N L} \frac{m_{i}}{N L} d\left(m_{i} s_{i} c_{i}\right) \exp \left(-\frac{1}{k_{B} T}\left(-h_{f} m_{i}-J s_{i}-L c_{i}\right)\right)}{\sum_{i=1, N L} d\left(m_{i} s_{i} c_{i}\right) \exp \left(-\frac{1}{k_{B} T}\left(-h_{f} m_{i}-J s_{i}-L c_{i}\right)\right)},
$$

where $N L$ is the number of distinct configuration of states $\langle m, s, c\rangle, d(m, s, c)$ is the number of configuration for a given set of values and where $h_{f}$ is given by the expression:

$$
h_{f}=-\left(\frac{\Delta-k_{B} T \ln g}{2}-G<\sigma>\right),
$$

This is a self consistent equation $\langle\sigma\rangle=f(\langle\sigma\rangle)$ that has been solved using the bisection technique. The $\Gamma$ interaction which has been often used by other authors in the mean field approximation [37] is equivalent to $\Gamma=q J+G$ where $q$ is the nearest neigbhors number.

\subsection{Monte Carlo Entropic Sampling}

We used the Monte Carlo entropic sampling (MCES) method [38] to generate a table that contains the dimensionless macroscopic variables, $m, s, c$, and their density, $d(m, s, c)$. Therefore, we used the principle of MCES described by Shteto et al. [39] which consists in introducing in the detailed balance equation a suited biased distribution $P$ in order to favor configurations belonging to weakly degenerate macrostates and to dampen those belonging to the highly degenerate macrostates.

The balance equation is given by:

$$
P_{i} W(i \rightarrow j)=P_{j} W(j \rightarrow i),
$$

and the biasing probability was chosen as the inverse of the desired restricted density of states.

$$
P_{i} \propto \frac{1}{d\left(m_{i}, s_{i}, c_{i}\right)},
$$


In this case, the balance equation can be written:

$$
\frac{W(i \rightarrow j)}{W(j \rightarrow i)}=\frac{P_{j}}{P_{i}}=\frac{d\left(m_{i}, s_{i}, c_{i}\right)}{d\left(m_{j}, s_{j}, c_{j}\right)}
$$

Since in the first Monte Carlo step the density of the state $d(m, s, c)$ is unknown, we put all $d(m, s, c)$ equal to 1 . So, after iteration $k$ the density will be $d_{k}(m, s, c)$. Then, using $d_{k}(m, s, c)$ as a bias, a MC sampling is run; it is termed a 'Monte Carlo stage' and yields a histogram of the frequency of the macrostates [38]: $H_{k}(m, s, c)$ :

$$
H_{k}(m, s, c) \propto d(m, s, c) \frac{1}{d_{k}(m, s, c)} .
$$

The resulting restricted density of states is obtained after applying a correction for the bias:

$$
d_{k+1}(m, s, c) \propto d_{k}(m, s, c) H_{k}(m, s, c) \text {. }
$$

After the table that consists from $m, s, c$, and $d(m, s, c)$ is obtained, the partition function can be calculated using the following expression:

$$
Z=\sum_{(m, s, c)} d(m, s, c) \exp (-\beta(-h m-J s-L c))
$$

and all the thermodynamic properties of the system can then be derived analytically.

\section{Results and Discussion}

The stepwise SCO behavior observed experimentally in 3D SCO systems [11] was modelled by Chiruta et al. [21] considering an antiferromagnetic-like short-range interaction, a ferromagnetic-like long-range interaction and considering the system bordered with metallic centers blocked in the HS state as well. The multi-step transitions obtained experimentally for $1 \mathrm{D}$ and 2D SCO systems $[12,13]$ were modelled by considering that the edge metallic centers interact with their local environment $[30,33]$ or by blocking the edge metallic centers in HS state $[31,32]$.

In this work, we first consider a 3D SCO system whose edge metallic centers interact with its environment and apply the bisection technique to equation (7). We derived the HS fraction, $\mathrm{n}_{\mathrm{HS}}$, from Equation (6). The SCO selected system contains 125 metallic centers $(5 \times 5 \times 5)$ of which 98 are surface metallic centers and only 27 are inner metallic centers. If we take into account that a typical distance between metal centers is of about $9 \AA$ [40] it means a cubic sampling of $3.6 \mathrm{~nm} \times 3.6 \mathrm{~nm} \times 3.6 \mathrm{~nm}$. As a result, a hysteretic multistep transition is predicted (Figure 2).

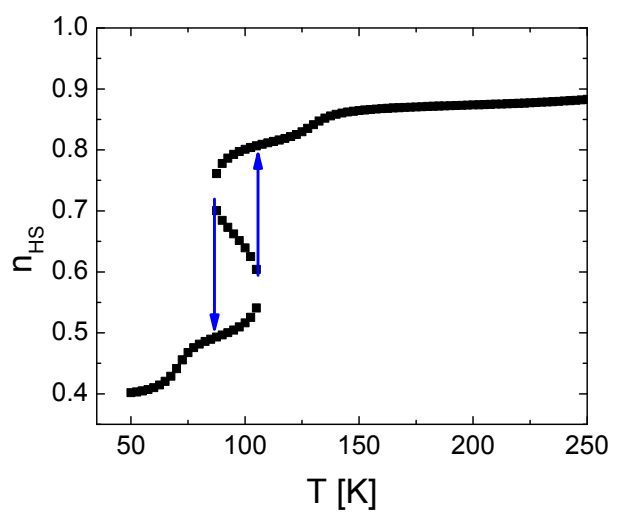

Figure 2. Simulated HS fraction, $\mathrm{n}_{\mathrm{HS}}$, as a function of temperature for a 3D SCO system. The computational parameters are $N=125(5 \times 5 \times 5), \Delta / k_{B}=1450 \mathrm{~K}\left(=1007.75 \mathrm{~cm}^{-1}\right), \mathrm{G} / \mathrm{k}_{B}=470 \mathrm{~K}$ $\left(=326.65 \mathrm{~cm}^{-1}\right), J / k_{B}=-100 \mathrm{~K}\left(=-69.5 \mathrm{~cm}^{-1}\right), L / k_{B}=750 \mathrm{~K}\left(=521.25 \mathrm{~cm}^{-1}\right)$ and $\ln (\mathrm{g})=4.7$. 
For comparison purposes, we plotted the computed thermal behavior without edge effect on Figure 3. The following conclusions can be drawn: (i) the hysteresis related to the middle-step disappears; (ii) the HS fraction increases from 0 (when $L / k_{B}=0 \mathrm{~K}$ ) to 0.4 (when $L / k_{B}=750 \mathrm{~K}$ ); (iii) the transition is shifted to lower temperatures, which means that the edge interaction seems to have an action opposite to a typical applied pressure because the edge interaction favors the HS state while an applied pressure is known to favor the LS state, due to its lower ionic volume.

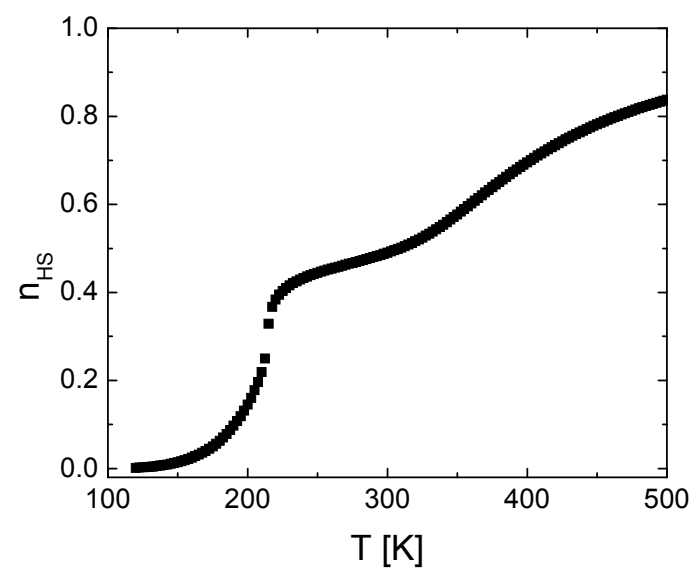

Figure 3. Simulated HS fraction, $\mathrm{n}_{\mathrm{HS}}$, as a function of temperature for a $3 \mathrm{D}$ spin crossover (SCO) system when $L / k_{B}=0 \mathrm{~K}$. Other parameters are the same as those of Figure 1.

The computed thermal behavior of a 2D SCO system (where the interactions within metallic centres between planes are less than $10 \%$ of the interaction between metallic centres in the same plane) with 121 metallic centers is shown in Figure 4. Even if the number of metallic centers is almost equal to the number of metallic centers of the 3D system used above (125), the role of edge effect is reduced, because the number of edge metallic centers is only 40 compared to 98 metallic centers in the case of the 3D system. The spin transition is still incomplete, proceeds in three steps but is no longer hysteretic.

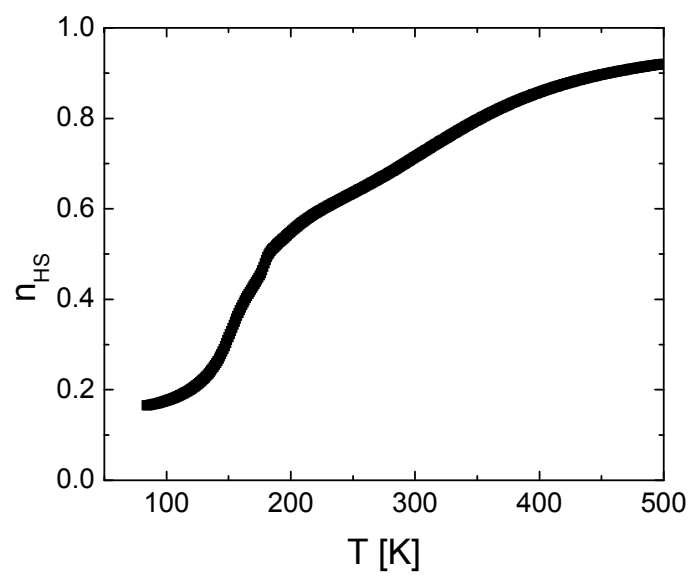

Figure 4. Simulated HS fraction, $\mathrm{n}_{\mathrm{HS}}$, as a function of temperature for a $2 \mathrm{D}$ SCO system with $N=121$ $(11 \times 11)$. Other parameters are the same as those of Figure 1 .

Finally, we have investigated the increase of the system size (Figure 5). By increasing the system size, ie by decreasing the ratio between edge and inner metallic centers (from 7 for a cube system with 64 metallic centers to 3.629 for a system with 125 metallic centers and to 2.375 for a system with 216 metallic centers), the edge effect decreases and the role of inner metallic centers increases. As a 
result, when we increase the size of the system, the equilibrium temperature, $T_{1 / 2}$, is shifted to higher temperatures and the transition proceeds more continuously from LS to HS.

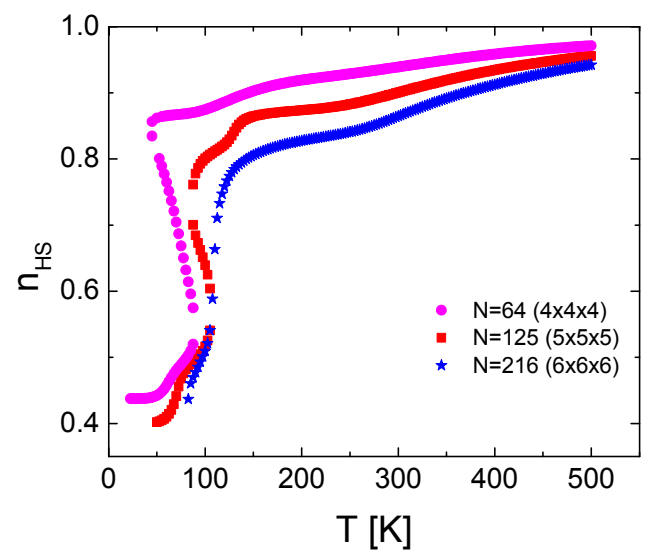

Figure 5. Simulated HS fraction, $\mathrm{n}_{\mathrm{HS}}$, as a function of temperature for 3D SCO systems of different sizes. Other parameters are the same as those of Figure 1.

\section{Conclusions}

In this communication, we have studied the role of the interaction between edge metallic centers and their local environment on the thermal behavior of spin crossover compounds. Our predictions show that: (i) it favors the high-spin state at the surface which means that it plays an equivalent role opposite to a typical applied pressure; (ii) it shifts the transition to lower temperatures and increases the residual high-spin fraction. As a result, we claim that the 3-step spin crossover originates from three interactions: (1) an interaction between edge-metallic center with the matrix; (2) a "ferromagnetic-like" long-range interaction and (3) an "antiferromagnetic-like" short-range interaction. This prediction is of the utmost importance in the current development of new spin crossover materials for smart applications.

Acknowledgments: This work was partially supported by Romanian National Authority for Scientific Research, CNCS-UEFISCDI (PN-II-RU-TE-2014-4-2695), a bilateral grant WBI-Romanian Academy, and the Fonds National de la Recherche Scientifique (FNRS) (PDR T.0102.15). Financial support from the project "Integrated Center for research, development and innovation in Advanced Materials, Nanotechnologies, and Distributed Systems for fabrication and control - MANSiD", Contract No. 671/09.04.2015, Sectoral Operational Program for Increase of the Economic Competitiveness co-funded from the European Regional Development Fund is also acknowledged. Funding from chair MSE (Materials Simulation and Engineering) of University of Versailles St Quentin en Yvelines is also acknowledged as well as COST actions MP1202 and CM1305.

Author Contributions: J.L. proposed the subjet; C.J. and J.L. performed the calculations and analyzed the data; C.J., J. L., A.R. and Y. G. wrote the paper and participated to all discussions.

Conflicts of Interest: The authors declare no conflict of interest. The founding sponsors had no role in the design of the study; in the collection, analyses, or interpretation of data; in the writing of the manuscript, and in the decision to publish the results.

\section{References}

1. Linares, J.; Codjovi, E.; Garcia, Y. Pressure and Temperature Spin Crossover Sensors with Optical Detection. Sensors 2012, 12, 4479-4492. [CrossRef] [PubMed]

2. Jureschi, C.M.; Rusu, I.; Codjovi, E.; Linares, J.; Garcia, Y.; Rotaru, A. Thermo- and piezochromic properties of $\left[\mathrm{Fe}(\right.$ hyptrz) $] \mathrm{A}_{2} \cdot \mathrm{H}_{2} \mathrm{O}$ spin crossover $1 \mathrm{D}$ coordination polymer: Towards spin crossover based temperature and pressure sensors. Physica B 2014, 449, 47-51. [CrossRef]

3. Jureschi, C.M.; Linares, J.; Rotaru, A.; Ritti, M.H.; Parlier, M.; Dîrtu, M.M.; Wolff, M.; Garcia, Y. Pressure sensor via optical detection based on a 1D spin transition coordination polymer. Sensors 2015, 15, 2388-2398. [CrossRef] [PubMed] 
4. Rotaru, A.; Dugay, J.; Tan, R.P.; Gural'skiy, I.A.; Salmon, L.; Demont, P.; Carrey, J.; Molnar, G.; Respaud, M.; Bousseksou, A. Nano-electromanipulation of Spin Crossover Nanorods: Towards Switchable Nanoelectronic Devices. Adv. Mater. 2013, 25, 1745-1749. [CrossRef] [PubMed]

5. Gütlich, P.; Hauser, A.; Spiering, H. Thermal and optical switching of iron(II) complexes. Angew. Chem. Int. Ed. Engl. 1994, 33, 2024-2054. [CrossRef]

6. Gütlich, P.; Garcia, Y.; Goodwin, H.A. Spin crossover phenomena in Fe(II) complexes. Chem. Soc. Rev. 2000, 29, 419-427. [CrossRef]

7. Gütlich, P.; Goodwin, H.A. Spin crossover-An overall perspective. In Spin Crossover in Transition Metal Compounds I; Springer Berlin Heidelberg: Berlin, Germay, 2004; Volume 233, pp. 1-47.

8. Garcia, Y.; van Koningsbruggen, P.J.; Lapouyade, R.; Fournes, L.; Rabardel, L.; Kahn, O.; Ksenofontov, V.; Levchenko, G.; Gütlich, P. Influences of temperature, pressure, and lattice solvents on the spin transition regime of the polymeric compound $\left[\mathrm{Fe}(\text { hyetrz })_{3}\right] \mathrm{A}_{2} \cdot 3 \mathrm{H}_{2} \mathrm{O}$, with hyetrz $=4$ - $(2$ '-hydroxyethyl $)-1,2,4$-triazole and $\mathrm{A}^{-}=3$-nitrophenylsulfonate. Chem. Mater. 1998, 10, 2426-2433. [CrossRef]

9. Real, J.A.; Gaspar, A.B.; Munoz, M.C.; Gütlich, P.; Ksenofontov, V.; Spiering, H. Bipyrimidine-Bridged Dinuclear Iron(II) Spin Crossover Compounds. In Spin Crossover in Transition Metal Compounds I; Springer Berlin Heidelberg: Berlin, Germany, 2004; Volume 233, pp. 167-193.

10. Ohba, M.; Yoneda, K.; Agusti, G.; Munoz, M.C.; Gaspar, A.B.; Real, J.A.; Yamasaki, M.; Ando, H.; Nakao, Y.; Sakaki, S.; et al. Bidirectional chemo-switching of spin state in a microporous framework. Angew. Chem. Int. Ed. 2009, 48, 4767-4771. [CrossRef] [PubMed]

11. Sciortino, N.F.; Scherl-Gruenwald, K.R.; Chastanet, G.; Halder, G.J.; Chapman, K.W.; Letard, J.F.; Kepert, C.J. Hysteretic Three-Step Spin Crossover in a Thermo- and Photochromic 3D Pillared Hofmann-type Metal-Organic Framework. Angew. Chem. Int. Ed. 2012, 51, 10154-10158. [CrossRef] [PubMed]

12. Kosone, T.; Tomori, I.; Kanadani, C.; Saito, T.; Mochida, T.; Kitazawa, T. Unprecedented three-step spin-crossover transition in new 2-dimensional coordination polymer $\left\{\mathrm{Fe}^{\mathrm{II}}(4 \text {-methylpyridine })_{2}\left[\mathrm{Au}-\mathrm{I}(\mathrm{CN})_{2}\right]_{2}\right]$. Dalton Trans. 2010, 39, 1719-1721. [CrossRef] [PubMed]

13. Nishi, K.; Kondo, H.; Fujinami, T.; Matsumoto, N.; Iijima, S.; Halcrow, M.A.; Sunatsuki, Y.; Kojima, M. Stepwise Spin Transition and Hysteresis of a Tetrameric Iron(II) Complex, facTris(2-methylimidazol-4-ylmethylidene-n-hexylamine) iron(II) Chloride Hexafluorophosphate, Assembled by Imidazole center dot center dot center dot Chloride Hydrogen Bonds. Eur. J. Inorg. Chem. 2013, 5-6, 927-933. [CrossRef]

14. Garcia, Y.; Kahn, O.; Rabardel, L.; Chansou, B.; Salmon, L.; Tuchagues, J.P. Two-step spin conversion for the three-dimensional compound tris(4,4'-bis-1,2,4-triazole)iron(II) diperchlorate. Inorg. Chem. 1999, 38, 4663-4670. [CrossRef] [PubMed]

15. Enachescu, C.; Constant-Machado, H.; Codjovi, E.; Linares, J.; Boukheddaden, K.; Varret, F. Direct access to the photo-excitation and relaxation terms in photo-switchable solids: Non-linear aspects. J. Phys. Chem. Solids 2001, 62, 1409-1422. [CrossRef]

16. Jeftic, J.; Menendez, N.; Wack, A.; Codjovi, E.; Linares, J.; Goujon, A.; Hamel, G.; Klotz, S.; Syfosse, G.; Varret, F. A helium-gas-pressure apparatus with optical-reflectivity detection tested with a spin-transition solid. Meas. Sci. Tech. 1999, 10, 1059-1064. [CrossRef]

17. Morscheidt, W.; Jeftic, J.; Codjovi, E.; Linares, J.; Bousseksou, A.; Constant-Machado, H.; Varret, F. Optical detection of the spin transition by reflectivity: application to $\left[\mathrm{Fe}_{\mathrm{x}} \mathrm{Co}_{1-x}(\mathrm{btr})_{2}(\mathrm{NCS})_{2}\right] \cdot \mathrm{H}_{2} \mathrm{O}$. Meas. Sci. Tech. 1998, 9, 1311-1315. [CrossRef]

18. Mishra, V.; Mukherjee, R.; Linares, J.; Balde, C.; Desplanches, C.; Letard, J.F.; Collet, E.; Toupet, L.; Castro, M.; Varret, F. Temperature-dependent interactions and disorder in the spin-transition compound $\left[\mathrm{Fe}-\mathrm{II}(\mathrm{L})_{2}\right]\left[\mathrm{ClO}_{4}\right]_{2} \cdot \mathrm{C}_{7} \mathrm{H}_{8}$ through structural, calorimetric, magnetic, photomagnetic, and diffuse reflectance investigations. Inorg. Chem. 2008, 47, 7577-7587. [CrossRef] [PubMed]

19. Weber, B.; Carbonera, C.; Desplanches, C.; Létard, J.F. Stepwise spin transition in a mononuclear iron(II) complex with unusually wide plateau. Eur. J. Inorg. Chem. 2008, 10, 1589-1598. [CrossRef]

20. Atitoaie, A.; Tanasa, R.; Enachescu, C. Size dependent thermal hysteresis in spin crossover nanoparticles reflected within a Monte Carlo based Ising-like model. J. Mag. Mag. Mater. 2012, 324, 1596-1600. [CrossRef]

21. Chiruta, D.; Linares, J.; Dimian, M.; Alayli, Y.; Garcia, Y. Role of Edge Atoms in the Hysteretic Behaviour of 3D Spin Crossover Nanoparticles Revealed by an Ising-Like Model. Eur. J. Inorg. Chem. 2013, 29, 5086-5093. [CrossRef] 
22. Chiruta, D.; Jureschi, C.M.; Linares, J.; Graur, A.; Dimian, M.; Rotaru, A. Analysis of Architecture Effect on Hysteretic Behavior of 3-D Spin Crossover Nanostructures. IEEE Trans. Magn. 2014, 50, 1-4. [CrossRef]

23. Boukheddaden, K.; Linares, J.; Codjovi, E.; Varret, F.; Niel, V.; Real, J.A. Dynamical Ising-like model for the two-step spin-crossover systems. J. App. Phys. 2003, 93, 7103-7105. [CrossRef]

24. Enachescu, C.; Tanasa, R.; Stancu, A.; Varret, F.; Linares, J.; Codjovi, E. First-order reversal curves analysis of rate-dependent hysteresis: The example of light-induced thermal hysteresis in a spin-crossover solid. Phys. Rev. B 2005, 72. [CrossRef]

25. Klokishner, S.; Linares, J.; Varret, F. Effect of hydrostatic pressure on phase transitions in spin-crossover 1D systems. Chem. Phys. 2000, 255, 317-323. [CrossRef]

26. Wajnflasz, J.; Pick, R. Low-spin-high-spin transitions in $\mathrm{Fe}^{2+}$ complexes. J. Phys. Colloques 1971, $32, \mathrm{C} 1$. [CrossRef]

27. Bousseksou, A.; Nasser, J.; Linares, J.; Boukheddaden, K.; Varret, F. Ising-like model for the 2-step spin-crossover. J. Phys. I 1992, 2, 1381-1403. [CrossRef]

28. Linares, J.; Spiering, H.; Varret, F. Analytical solution of 1D Ising-like systems modified by weak long range interaction - Application to spin crossover compounds. Eur. Phys. J. B 1999, 10, 271-275. [CrossRef]

29. Chiruta, D.; Linares, J.; Dahoo, P.R.; Dimian, M. Analysis of long-range interaction effects on phase transitions in two-step spin-crossover chains by using Ising-type systems and Monte Carlo entropic sampling technique. J. Appl. Phys. 2012, 112, 7. [CrossRef]

30. Chiruta, D.; Jureschi, C.M.; Linares, J.; Dahoo, P.; Garcia, Y.; Rotaru, A. On the origin of multi-step spin transition behaviour in 1D nanoparticles. Eur. Phys. J. B 2015, 88, 1-5. [CrossRef]

31. Chiruta, D.; Linares, J.; Dimian, M.; Garcia, Y. Size Effect and Role of Short- and Long-Range Interactions on 1D Spin-Crossover Systems within the Framework of an Ising-Like Model. Eur. J. Inorg. Chem. 2013, 5-6, 951-957. [CrossRef]

32. Chiruta, D.; Linares, J.; Garcia, Y.; Dimian, M.; Dahoo, P.R. Analysis of multi-step transitions in spin crossover nanochains. Physica B. 2014, 434, 134-138. [CrossRef]

33. Jureschi, C.M.; Pottier, B.L.; Linares, J.; Richard Dahoo, P.; Alayli, Y.; Rotaru, A. Simulation of multi-steps thermal transition in 2D spin-crossover nanoparticles. Physica B 2016. [CrossRef]

34. Chiruta, D.; Jureschi, C.M.; Linares, J.; Garcia, Y.; Rotaru, A. Lattice architecture effect on the cooperativity of spin transition coordination polymers. J. Appl. Phys. 2014, 115, 053523. [CrossRef]

35. Peng, H.; Tricard, S.; Félix, G.; Molnár, G.; Nicolazzi, W.; Salmon, L.; Bousseksou, A. Re-Appearance of Cooperativity in Ultra-Small Spin-Crossover $\left[\mathrm{Fe}(\mathrm{pz})\left\{\mathrm{Ni}(\mathrm{CN})_{4}\right\}\right.$ Nanoparticles. Angew. Chem. 2014, 126, 11074-11078. [CrossRef]

36. Muraoka, A.; Boukheddaden, K.; Linares, J.; Varret, F. Two-dimensional Ising-like model with specific edge effects for spin-crossover nanoparticles: A Monte Carlo study. Phys. Rev. B 2011, 5, 84. [CrossRef]

37. Spiering, H. Elastic interaction in spin crossover compounds. In Spin Crossover in Transition Metal Compounds III; Springer: Berlin Heidelberg, Germany, 2004; Volume 235, pp. 171-195.

38. Linares, J.; Enachescu, C.; Boukheddaden, K.; Varret, F. Monte Carlo entropic sampling applied to spin crossover solids: the squareness of the thermal hysteresis loop. Polyhedron 2003, 22, 2453-2456. [CrossRef]

39. Shteto, I.; Linares, J.; Varret, F. Monte Carlo entropic sampling for the study of metastable states and relaxation paths. Phys. Rev. E 1997, 56, 5128-5137. [CrossRef]

40. Pillet, S.; Hubsch, J.; Lecomte, C. Single crystal diffraction analysis of the thermal spin conversion in $\left[\mathrm{Fe}(\mathrm{btr})_{2}(\mathrm{NCS})_{2}\right]\left(\mathrm{H}_{2} \mathrm{O}\right)$ : Evidence for spin-like domain formation. Eur. Phys. J. B 2004, 38, 541-552. [CrossRef]

(C) 2016 by the authors; licensee MDPI, Basel, Switzerland. This article is an open access article distributed under the terms and conditions of the Creative Commons by Attribution (CC-BY) license (http://creativecommons.org/licenses/by/4.0/). 